\title{
Ámbitos de uso del Mapudungun en comunidades PeWenches y LafKenches de la Región del Bío-Bío *
}

\author{
Marisol Henríquez Barahona**
}

\begin{abstract}
Resumen
Este trabajo tiene como propósito identificar y analizar algunos de los principales ámbitos o dominios sociales de uso del mapudungun en comunidades pewenches y lafkeches de la región del Bío-Bío. La muestra estuvo conformada por un grupo de 40 escolares mapuches de entre 12 y 15 años de edad, que se encontraban cursando $7^{\circ}$ y $8^{\circ}$ año básico en escuelas rurales pertenecientes a las Comunas de Tirúa y Alto Bío-Bío. Para la recolección del material se utilizó un cuestionario sociolingüístico y una entrevista focalizada que se aplicó a los escolares y a los educadores tradicionales, respectivamente. A través del análisis de los datos se evidencian los principales ámbitos de uso intracomunitarios en los que actualmente la lengua tradicional predomina y aquéllos, donde el castellano ha ido ampliando su campo de acción. Este análisis permite, además, aportar antecedentes sociolingüísticos sobre el estado del mapudungun en estas zonas rurales de contacto.
\end{abstract}

Palabras clave: Lenguas en contacto - ámbitos de uso - mapudungun - castellano desplazamiento lingüístico.

\section{Domains of Mapudungun use un Pewenches and LAFKenChes COMmunities Bío Bío Región}

\begin{abstract}
This paper aims at identifying and analyzing some of the main areas or social domains in the use of Mapudungun in Pewenches and Lafkenches communities from Bío- Bío region. The sample was made up of 40 Mapuche students, from rural schools, whose age ranged between 12 and 15. A questionnaire and a focused interview were used to collect data. These instruments were applied to students and teachers respectively. The data analysis shows the main domains of Mapudungun use inside each community and also the domains of Spanish use in communities where this language is gaining ground. This analysis may also provide some sociolinguistic background of the state of Mapudungun in these rural areas.
\end{abstract}

Keywords: Languages ??in contact- sociolinguistics domains -mapudungun- Spanishlanguage shift

Recibido: 29-09- 2014

Aceptado: 24-12-2014

* Este artículo está asociado a la tesis doctoral Vitalidad fonológica del madungun en escolares mapuches pewenches y lafkenches de la VIII región del Bio-Bio, Universidad de Concepción, Chile.

** Chilena. Doctora en Lingüística de la Universidad Católica de la Santísima Concepción, Concepción, Chile.mhenriquez@ucsc.cl 


\section{Introducción}

El concepto dominio de uso o dominio sociolingüístico, desarrollado inicialmente por Fishman (1971, 1982), se concibe como una abstracción o conjunto de situaciones comunicativas prototípicas, que giran en torno a determinados temas y situaciones, al tiempo que estructuran la percepción que los propios hablantes poseen de esos mismos contextos (Fishman, 1982). Este modelo ha sido de utilidad para sistematizar el proceso de funcionamiento de las elecciones lingüísticas en los contextos bilingües, aunque resulta insuficiente para explicar a cabalidad este proceso, pues se sabe que cuando un hablante elige una lengua y no otra, lo hace de acuerdo a una serie de normas y valores no estrictamente lingüísticos, sino sociales, culturales y psicológicos (Serrano, 2011).

El dominio o ámbito de uso adopta la organización social como base para la descripción de las elecciones lingüísticas. Cuando los hablantes emplean dos o más lenguas, es poco frecuente y hasta extraño que lo hagan en cualquier contexto. La elección de una $u$ otra lengua o variedad viene condicionada por ciertos factores sociales implicados en este proceso, entre los que sobresalen los sentimientos de solidaridad e identidad grupal, o los atributos que configuran la situación comunicativa (tema, estatus y caracteres personales de los participantes, lugar, etc), entre otros (Blas Arroyo, 2008; Serrano, 2011).

Fishman (1982) plantea la utilidad de los dominios de conducta individuales que se asocian comúnmente con una variedad o lengua en particular. Así, por ejemplo, a través de sus investigaciones, descubrió que en muchas comunidades bilingües, dominios como la escuela, iglesia, esfera laboral superior y gobierno son concordantes con una lengua o variedad " $\mathrm{A}$ " (variedad o lengua mayoritaria o de mayor prestigio), mientras que otros, como la familia, vecindad y esfera laboral inferior son concordantes con la variedad o lengua " $\mathrm{B}$ " (variedad o lengua minoritaria o de menor prestigio). Sin embargo, cada comunidad de habla tendrá o desarrollará sus propios dominios de uso para cada lengua o variedad disponible. Esto quiere decir que ningún conjunto fijo de dominios puede formularse a priori para todas las comunidades, puesto que "el conjunto de actividades que constituirá una agrupación de propósito, relaciones de roles y marco será específico de una cultura" (Saville Troike, 2005, p. 58). 
Los dominios o ámbitos de uso no sólo son útiles para la descripción funcional de las comunidades que experimentan variación socialmente estructurada en la "conversación", es decir, para el estudio de la elección lingüística, sino también para el probable y consecuente proceso de desplazamiento lingüístico que pudiera afectar a alguna de las dos lenguas en contacto (cf. Fishman, 1982; Blas Arroyo, 2008). La funcionalidad de una lengua minoritaria como medio de comunicación en diferentes ámbitos de uso es un factor social de gran relevancia en la explicación de los fenómenos de cambio y en la proyección de la lengua, ya que en los dominios íntimos y familiares se concreta el traspaso intergeneracional que permitiría el mantenimiento lingüístico. Es por esto que, en el estudio de lenguas en contacto, especialmente, si alguna de ellas se encuentra en proceso de retracción, es importante relevar y analizar los principales ámbitos que determinan el uso de una $\mathrm{u}$ otra lengua entre los hablantes nativos $\mathrm{y}$, a partir de ello, entregar insumos sobre el futuro de las lenguas en zona de contacto.

\section{2. Ámbito de uso y mantenimiento/desplazamiento lingüístico}

Para los especialistas, el ámbito de uso cumple un rol no menor en los procesos de mantenimiento y desplazamiento lingüístico (Fasold, 1996; Blas Arroyo, 2008; Serrano, 2011; Crystal, 2001 y Gimeno y Gimeno, 2003). Así, por ejemplo, si una lengua minoritaria pierde terreno en alguno de los dominios de uso que tradicionalmente le correspondían, se dice que estaría experimentando un proceso de desplazamiento: "en el momento en que una comunidad comienza a elegir una lengua en ámbitos o dominios en los que tradicionalmente se ha utilizado otra, la sustitución de la lengua está en curso (Moreno Fernández, 2005, p. 243). Esto quiere decir que la elección de una lengua en determinados ámbitos y no en otros, repercute directamente en el mantenimiento de un sistema: mientras más dominios de uso, mayor vitalidad lingüística (Fasold, 1996; Blas Arroyo, 2008; Serrano, 2011).

El resultado del proceso de competencia entre las lenguas en determinados ámbitos de uso generalmente se da en favor de la lengua de mayor prestigio. Para Siguan (2001), "la lengua del grupo dominante tiende a expansionarse, bien como consecuencia de una presión directa, bien simplemente como resultado del mayor prestigio que le concede 
el ser la lengua del grupo más poderoso" (Siguán, 2001, p. 258). La extensión o filtración de la nueva lengua en un ámbito determinado es un proceso gradual: la lengua débil va progresivamente cediendo espacios a la de mayor prestigio. Una lengua minoritaria nunca es reemplazada de repente por la lengua mayoritaria, sino que el uso lingüístico se hace variable, es decir, ambas lenguas en contacto se usan en el mismo contexto social o ámbito; después del estado de uso variable, el uso de la lengua minoritaria podría ser excluido (Appel y Muysken 1996, p. 63).

Cuando una lengua deja de usarse en ámbitos íntimos y familiares, se afecta la transmisión intergeneracional. Estehecho repercute directamente en el desempeño lingüístico y comunicativo de las generaciones más jóvenes de la comunidad (Fasold, 1996). Es decir, cuando la lengua minoritaria se mantiene en el dominio familiar se generaliza entre los hablantes más jóvenes, por el contrario, cuando se restringen sus posibilidades de uso se contribuye a su desplazamiento (Serrano, 2011).

La restricción en el uso de la lengua a ciertos contextos o dominios sociales y el quiebre de la transmisión intergeneracional tienen consecuencias, no sólo en la competencia lingüística de los hablantes, como ya se señaló, sino también en el plano formal del sistema, es decir, la disminución de ámbitos de uso trae consigo pérdida lingüística (Dorian, 1977, 1980; Andersen, 1982; Appel y Muysken, 1996; Grinevald, 1997; Crystal, 2001; Gimeno y Gimeno, 2003; Serrano, 2011). En este sentido, Crystal advierte que con cada pérdida de un campo o ámbito de uso de una lengua se produce no sólo una pérdida de vocabulario y de modelos de discurso, sino también pérdida de orden estilístico (2001). Esto ocurriría porque "una lengua o variedad minoritaria usada en menos situaciones comunicativas se vuelve más estática y no exhibe todas sus posibilidades de uso (Serrano, 2011, p. 317). En cambio, la expansión de funciones está estrechamente relacionada con la promoción y extensión de su estructura lingüística.

En definitiva, la pérdida o reducción de ámbitos de uso tiene consecuencias significativas no sólo en el mantenimiento de una lengua minoritaria, sino en los procesos de pérdida lingüística estructural. En esta misma línea, incluso, hay quienes plantean que la disminución de ámbitos tendría mayor peso que los factores estrictamente estructurales en el proceso de cambio y desplazamiento lingüístico por contacto: "la reducción del número de ámbitos de uso de una lengua parece ser una 
causa más fuerte de pérdida de variantes semántico-pragmáticas que la opción preferencial por una estructura paralela en la lengua de contacto (Gimeno y Gimeno, 2003, p. 99).

En resumen, la funcionalidad de una lengua minoritaria como medio de comunicación en diferentes ámbitos de uso es un factor social de gran relevancia en la explicación de los fenómenos de cambio, como el desplazamiento lingüístico o en el mantenimiento lingüístico, por ello su abordaje en una lengua minoritaria y minorizada como el mapudungun ${ }^{1}$ debe considerarse de relevancia, especialmente, si se pretende, a partir del análisis de los ámbitos, inferir evidencia sobre el estado actual de la lengua indígena en las comunidades focalizadas.

\section{La investigación}

\subsection{Objetivo}

El objetivo central de este trabajo es identificar y analizar los principales ámbitos o dominios sociales de uso del mapudungun en comunidades pewenches y lafkeches de la región del Bío-Bío, a partir de las declaraciones de los escolares y los educadores tradicionales pertenecientes a cuatro escuelas rurales ubicadas en zona de contacto.

\subsection{Metodología}

\subsubsection{Zona de estudio}

Para la realización de este trabajo seleccionamos cuatro comunidades o localidades mapuches rurales de la Octava Región: las comunidades cordilleranas de Cauñicu y Butalelbun, ubicadas en el valle del Queuco en Alto Bío-Bío y las localidades de Primer Agua y Ranquilhue, en la comuna costera de Tirúa.

\subsubsection{Los participantes}

Para el logro de nuestros objetivos, trabajamos con una muestra selectiva e intencionada de 40 escolares mapuches (20 niños de Tirúa y 20, de Alto Bío-Bío), de entre 12 y 15 años de edad, que se encontraban

1 Los escolares de Alto Bío-Bío llaman a su lengua chedungun y los de Tirúa, la denominan indistintamente chedungun y mapudungun. En este artículo utilizaremos ambas denominaciones para referirnos a la lengua mapuche. 
cursando $7^{\circ}$ y $8^{\mathrm{a}}$ año básico en cuatro escuelas rurales adscritas al Programa de Educación Intercultural Bilingüe. Esta muestra se complementó con la participación de cuatro informantes expertos (Hernández et al., 2003). Nos referimos a los Educadores tradicionales ${ }^{2}$ de cada establecimiento educacional seleccionado quienes tuvieron un rol complementario en esta investigación, pues, a través de entrevistas sociolingüísticas focalizadas, nos aportaron información de relevancia sobre los niños, sus familias y la comunidad en general.

\subsubsection{Recolección de la información}

Para la elicitación de los datos diseñamos y aplicamos un cuestionario sociolingüístico a los escolares y una entrevista a los educadores tradicionales. Este material sociolingüístico se complementó con las observaciones y registros in situ que realizó la investigadora durante el trabajo de campo en ambas zonas de estudio.

\section{Resultados}

A continuación se presentarán los resultados obtenidos en relación con los ámbitos de uso del mapudungun en cada comunidad, es decir, en qué contextos intracomunitarios se habla la lengua y cuáles son los eventos preferidos por los miembros de la comunidad para su uso.

\section{1. Ámbitos de uso del mapudungun en la comunidad: Alto Bío-Bío y Tirúa}

La primera pregunta que realizamos a los estudiantes sobre esta dimensión se refiere a la frecuencia de uso de la lengua originaria en siete ámbitos o dominios de uso: (1) Casa; (2) Escuela; (3) Posta; (4) Sede comunitaria; (5) Micro; (6) Campo; (7) Caminos. Específicamente, se preguntó "¿En qué lugares se habla lengua mapuche en tu comunidad y con qué frecuencia?". Para esta pregunta las opciones de respuestas eran: Siempre, Frecuentemente, A veces, Nunca. En el Gráfico 1 se puede observar los lugares de uso del mapudungun y su frecuencia en la zona de Alto Bío-Bío; y en el gráfico 2, los de Tirúa. Se presenta la información por zona, puesto que el objetivo es mostrar un panorama general de los

2 El educador tradicional es quien pone en práctica el proceso de enseñanza de la lengua indígena, junto al profesor mentor. Es necesario precisar que la figura del Educador tradicional aparece con la entrada en vigencia del nuevo subsector de Lengua Indígena en el curriculum escolar de enseñanza básica. 
usos; sin embargo, cuando sea necesario, se precisarán las principales diferencias internas entre las comunidades de cada sector.

\section{Gráfico 1}

Ámbitos de uso del mapudungun en Alto Bío-Bío (Cauñicu y Butalelbun)

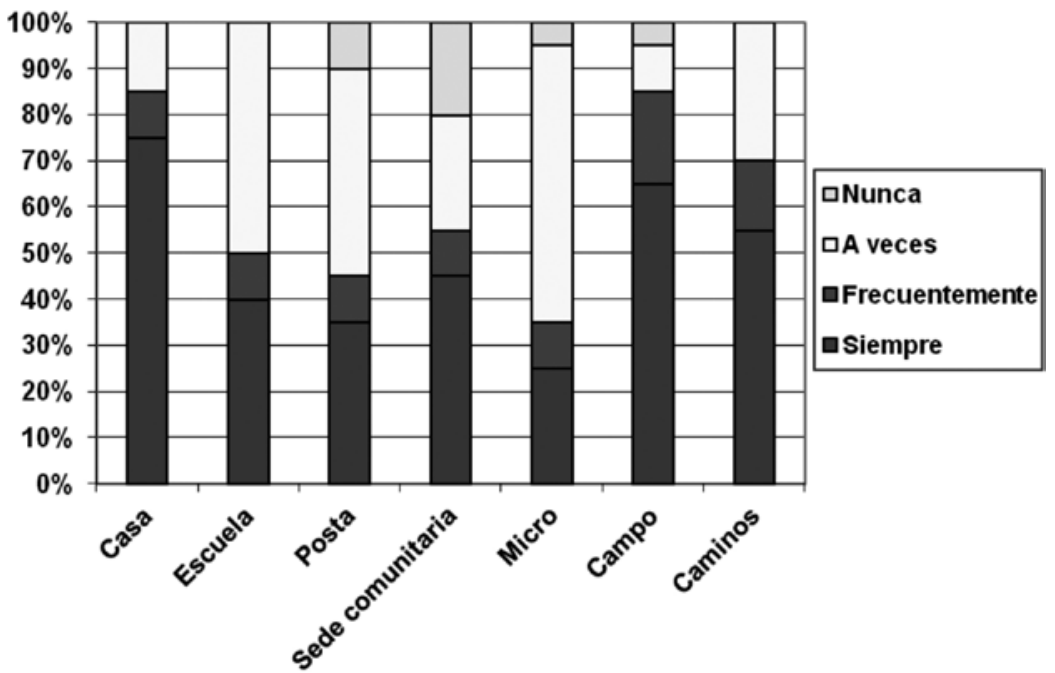

En las localidades de Alto Bío-Bío, analizadas en conjunto, se aprecia un alto porcentaje de uso (Siempre) del mapudungun en la casa $(75 \%)$, en el campo $(65 \%)$ y en los caminos (55\%), lo que evidencia que esta lengua se asocia primordialmente con los dominios de familia y amistad, que se correlacionan con un conjunto de valores de intimidad. La casa representa el hogar, las conversaciones familiares e íntimas; por su parte, los espacios camino y campo son, también, ámbitos intracomunitarios tradicionales donde se llevan a cabo conversaciones informales entre los miembros de la comunidad (vecinos, familiares y amigos) y están asociados con interacciones lingüísticas informales de intimidad y solidaridad. Ahora bien, los ámbitos donde el empleo es variable son la micro (60\% "A veces"), posta (45\% "A veces") y escuela ( $50 \%$ "A veces"). Estos son espacios públicos intracomunitarios en los que a menudo se dan cita miembros de comunidades diferentes y que se asocian con valores externos a la comunidad, pues se trataría de dominios no tradicionales de incorporación posterior. En el caso de la escuela, además, se trata de un contexto que evidencia estatus (Fishman, 1982). Todo esto, motiva la variabilidad y la tendencia al mayor empleo de la lengua de prestigio $u$ oficial. 
Otro factor a considerar es que en estos últimos escenarios ocurren, principalmente, interacciones entre interlocutores pewenches y nopewenches (profesor ${ }^{3}$, conductor ${ }^{4}$, auxiliar, enfermera, matrona y paramédicos, etc.), hecho que condiciona el uso de uno u otro código. Esto es relevante, porque la elección de una u otra lengua en comunidades bilingües no depende sólo del lugar o contexto social, sino de una serie compleja de otros factores, como el interlocutor, el tema y el grado de conocimiento de ambas lenguas, etc.

Si analizamos los datos desagregadamente en el sector, las diferencias más significativas que se observan entre ambas comunidades se dan en los ámbitos casa y escuela. Así, por ejemplo en Butalelbun, los escolares declaran que el chedungun se habla Siempre en la casa en más de un $90 \%$, en cambio en Cauñicu, el porcentaje desciende a menos de un $60 \%$. Lo mismo sucede en la escuela, donde la opción A veces asciende a más de un $85 \%$ en Cauñicú, mientras que en Butalelbun es la opción Siempre la preferida por los niños con más de un 70\%. En Cauñicu, la alternativa Siempre para este escenario no fue seleccionada por ningún estudiante. En efecto, en nuestras observaciones in situ apreciamos algunas diferencias en el uso de la lengua en la escuela por parte de los escolares de ambas comunidades. Así, en Butalelbun, el uso del chedungun es generalizado entre los niños. Se habla en todo momento, en circunstancias informales e íntimas al interior del recinto escolar: en el recreo, en el almuerzo, en conversaciones de pasillo entre alumnos, entre docentes y entre docentes y alumnos; incluso al interior de la sala de clases, cuando los estudiantes dialogaban entre ellos, la lengua tradicional surgía de manera espontánea, sin importar la presencia de la investigadora. En la escuela de Cauñicu, en cambio, no observamos el mismo panorama sociolingüístico, pues es la lengua castellana la que ocupaba la mayor parte de las instancias informales entre los niños y la alternancia de códigos sólo se observaba en las clases de Lengua Indígena. Al parecer, los escolares de esta escuela

3 En Cauñicu, observamos que la mayoría de los docentes que trabajaban en la escuela no eran pewenches, ni hablantes. Sólo una profesora, procedente de la Araucanía, era mapuche hablante y, justamente, era quien tenía a cargo, junto al Educador tradicional, la programación e implementación de las clases de Lengua Indígena. En Butalelbun, en cambio, la mayoría de los docentes era pewenche-hablante, a excepción de las profesoras de Lenguaje y Comunicación y de Educación Diferencial.

4 En el bus, que tuvimos la oportunidad de utilizar en muchas ocasiones para trasladarnos a la zona, observamos, en algunas oportunidades, el uso alternado del mapudungun y el castellano, aunque predominaba el castellano en la mayoría de las conversaciones entre los pasajeros; salvo en las interacciones comunicativas entre hablantes mayores. Durante nuestros viajes nunca observamos que los conductores o los auxiliares usaran el chedungun, aunque varios de ellos eran pewenches. 
que hablan chedungun lo hacen preferentemente con sus familiares en el hogar (Henríquez, 2013; 2014).

\section{Gráfico 2}

Ámbitos de uso del mapudungun en Tirúa (Primer Agua y Ranquilhue)

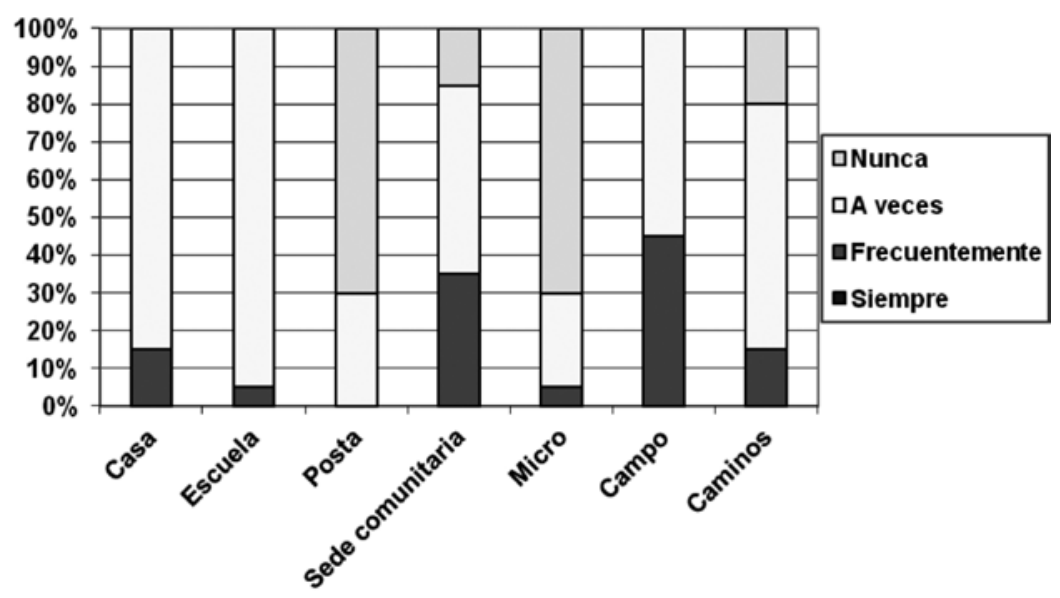

En Tirúa, como se observa en el gráfico 2, la categoría "Siempre" no fue seleccionada para ninguno de los ámbitos propuestos, evidenciando que, en ambas comunidades, el uso exclusivo de la lengua originaria no ocurre en ninguno de estos dominios. La opción "Frecuentemente", sí aparece en la esfera tradicional informal, pero con bajos índices: casa (15\%), campo (45\%) y caminos (15\%). Lo mismo, en los espacios públicos: escuela y micro $(5 \%)$ y sede comunitaria (35\%). Al respecto, cabe destacar que este uso "frecuente" del mapudungun en estos espacios comunitarios ocurre principalmente entre interlocutores ancianos o mayores, a excepción de la escuela, donde esta acción está en manos del educador tradicional y del profesor mentor.

La categoría "A veces" es la que mayoritariamente han seleccionado los escolares para indicar el uso esporádico del mapudungun en los diferentes lugares, lo que evidencia el predominio de elecciones lingüísticas variables. Así, por ejemplo, sucede en espacios tradicionales como la casa $(85 \%)$, campo $(55 \%)$ y caminos $(65 \%)$, donde la alternancia mapudungun-castellano pareciera ser la situación más característica. Por otra parte, de los espacios intracomunitarios no tradicionales, la posta es donde menos se habla el mapudungun ( $30 \%$ A veces y $70 \%$ Nunca), lo que se explica no sólo porque en este escenario se reúnen interlocutores 
mapuches y no-mapuches, sino porque, además, éste es un espacio público donde se trata y se habla del tema "salud" desde una perspectiva "occidental" (etiología, diagnóstico y tratamiento). De seguro, otra sería la categoría seleccionada para caracterizar el tipo de interacción comunicativa, si se diera en el contexto de un "machitun", es decir, si se acudiera a la "machi" para el tratamiento de una enfermedad. Algo similar ocurre en otro espacio público no tradicional como es la micro (bus), donde, según los escolares, los mapuches prefieren no emplear el mapudungun (70\% "Nunca").

No nos llama la atención que la lengua originaria se emplee en la escuela, aunque sólo sea "A veces" (95\%), puesto que en esta institución se está implementado el Programa EIB y, por ende, el subsector de Lengua Indígena, por lo que la enseñanza de la lengua y cultura mapuche es parte del curriculum escolar.

A diferencia de lo ocurrido en el sector cordillerano, el panorama sociolingüístico observado en esta zona es bastante homogéneo, es decir, los datos no evidencian diferencias importantes entre Ranquilhue y Primer Agua, en relación con los ámbitos de uso del mapudungun.

\section{2 Ámbitos de uso y eventos comunicativos}

La segunda pregunta que efectuamos a los escolares se relaciona con la frecuencia de uso de la lengua originaria en siete eventos o instancias comunicativas que se realizan en la comunidad: (1) Conversaciones familiares en general; (2) Conversaciones de ancianos (3) Ngillatun; (4) Wetripantü; (5) Fiestas y celebraciones; (6) Trabajo; (7) Clases. Específicamente, se preguntó "¿En qué situaciones se habla la lengua mapuche en tu comunidad y con quéfrecuencia?". Aligual que en la pregunta anterior, las opciones de respuestas fueron: Siempre, Frecuentemente, A veces, Nunca. Estos eventos comunicativos se actualizan en algunos de los espacios o lugares anteriormente señalados, es decir, son parte de los ámbitos de uso. Así, por ejemplo, las Conversaciones familiares en general ocurren principalmente en el dominio casa, mientras que las clases, se dan en la escuela, etc.

Al igual que para la pregunta anterior, presentamos los resultados por zona. Así, en el Gráfico 3 se observan las frecuencias de uso del mapudungun, para Alto Bío-Bío, en el contexto de los eventos comunicativos antes señalados. 


\section{Gráfico 3}

Uso del mapudungun en siete eventos comunicativos (Butalelbun y Cauñicu)

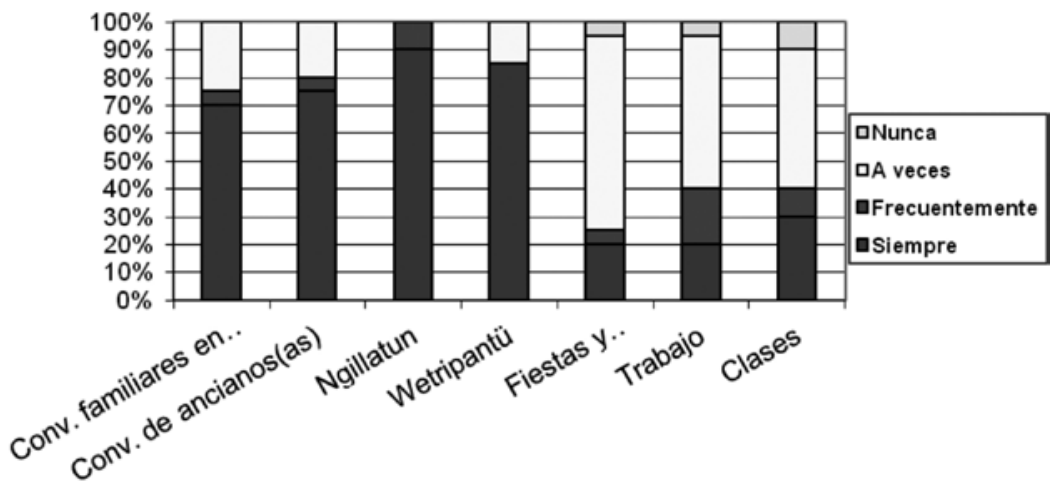

En Butalelbun y Cauñicu destaca el hecho de que los eventos comunicativos en los cuales se emplea mayoritariamente el mapudungun ("Siempre") son las Conversaciones familiares en general (70\%), Conversaciones de ancianos(as) (75\%), Ngillatun (90\%) y Wetripantü (85\%), mientras que el uso exclusivo de esta lengua disminuye drásticamente en los eventos Fiestas y celebraciones (20\%), Trabajo (20\%) y Clases (30\%). Esto coincide con los resultados de la pregunta anterior, y confirma que el chedungun se emplea mayoritariamente en la esfera familiar e íntima y, por cierto, en el ámbito ceremonial, mientras que su difusión social es menor en los espacios públicos.

Como ya se señaló, existen situaciones comunicativas en las que algunos factores contextuales pueden dar lugar a elecciones lingüísticas variables, donde la selección de una u otra lengua puede darse ya sea considerando como elemento preferente el escenario, o bien el tipo de relación entre los interlocutores ${ }^{5}$. Esto es lo que sucede en los eventos comunicativos públicos formales o informales que ocurren al interior de la comunidad, en los cuales la categoría " $A$ veces" es la preferente, lo que muestra el uso alternado de ambos códigos (fiestas y celebraciones $(70 \%)$, trabajo $(55 \%)$ y clases $(50 \%)$.

5 Mientras aplicábamos el cuestionario sociolingüístico a los escolares de Butalelbun al interior de la sala de clases, un niño se dirige a su compañero en mapudungun para comentar las preguntas del instrumento. En este caso los alumnos eligen esta lengua, dado que la relación entre los dos interlocutores es de intimidad y solidaridad, pese a encontrarse en un ámbito formal y con presencia de la investigadora en la escena. 


\section{Gráfico 4}

Uso del mapudungun en siete eventos comunicativos (Primer Agua y Ranquilhue)

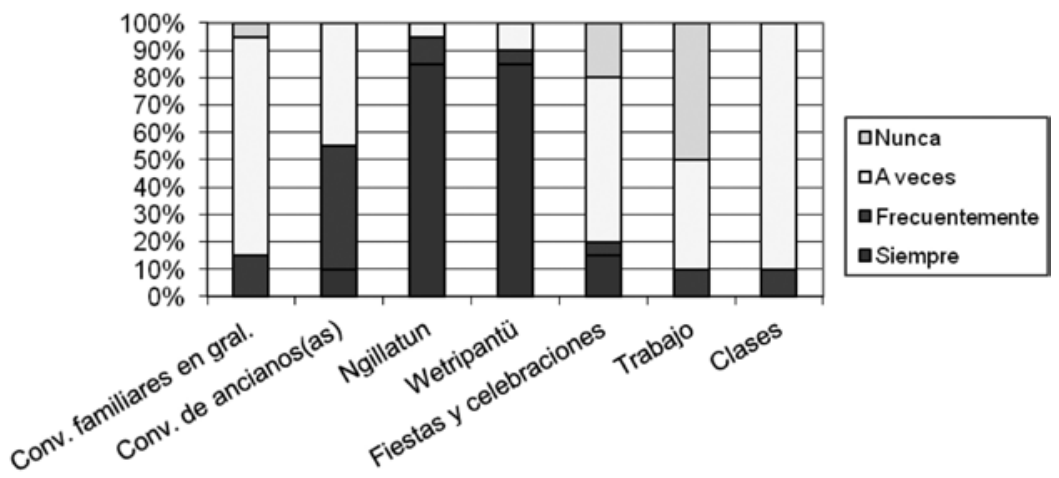

En Tirúa, se observa que la fidelidad al chedungun está principalmente asociada a dos eventos comunicativos: el ngillatun (85\% "Siempre") y el wetripantü (85\% "Siempre"); únicos reductos sociales donde el uso de esta lengua aún es ampliamente predominante. Estos datos coinciden con los obtenidos por Olate et al., en la zona lafkenche de Isla Huapi en la IX región de la Araucanía, donde el ámbito ceremonial se releva como el de mayor uso de la lengua ancestral (2013).

En la esfera doméstica y familiar, cuando los interlocutores son ancianos, el uso de la lengua todavía se mantiene vigente $(10 \%$ "Siempre" y 45\% "Frecuentemente"), pero en ningún caso comparable con ambas comunidades de Alto Bío-Bío, donde las Conversaciones de ancianos(as) se caracterizan por el empleo casi exclusivo de la lengua minoritaria. Siguiendo con el análisis en el mismo ámbito doméstico, en las Conversaciones familiares en general se observa que el uso de la lengua es menor (0\% "Siempre", 15\% "Frecuentemente" y 80\% "A veces"). Esto, porque el castellano ha penetrado de manera muy significativa en el ámbito familiar, restringiendo severamente el empleo del vernáculo. Las consecuencias de esta situación son relevantes, porque, como se sabe, uno de los escenarios más habituales del desplazamiento lingüístico corresponde al quiebre de la transmisión generacional entre padres e hijos (Fasold, 1996; Blas Arroyo, 2008). De ahí que la presencia del castellano en los dominios domésticos -especialmente en el hogar, donde se concreta la transmisión- constituye uno de los factores más explicativos del alto grado de abandono de la lengua ancestral en estas comunidades. 
En definitiva, fuera de la esfera ceremonial y de las interacciones entre ancianos, la lengua indígena está siendo reemplazada por el castellano o aparece en alternancia con ésta. Así sucede, por ejemplo, en eventos comunicativos tales como: Fiestas y celebraciones (60\% "A veces", $20 \%$ "Nunca"), Trabajo (40\% "A veces", 50\% "Nunca") y Clases (90\% "A veces"), donde el cambio al castellano parece rápido y constante.

En resumen, una mirada general a los eventos comunicativos nos revela que en esta zona el uso del castellano se ha hecho un camino en las funciones informales, en los dominios familiares del hogar y comunitarios públicos, desplazando a la lengua que tradicionalmente se usaba en estos ámbitos. A su vez, el mapudungun se ha ido relegando a aquellas situaciones altamente ceremoniales o rituales ${ }^{6}$ como el ngillatun y el wetripantu, aunque se debe destacar que actualmente ha encontrado cierto espacio de uso en otras funciones formales anteriormente inexistentes, como en los dominios educativos.

A continuación presentamos algunas afirmaciones realizadas por los educadores tradicionales que vienen a complementar y corroborar lo señalado por los estudiantes en relación con los actuales usos de la lengua en la comunidad.

\section{Declaraciones del educador tradicional}

Localidad

"En todo momento se habla chedungun, diariamente, en la casa, en Butalelbun la posta, en los caminos, en la micro [...] castellano hablan cuando hay reuniones, si algo tenemos que discutir hablamos castellano o chedungun".

“En la escuela se habla chedungun y castellano, pero la lengua que Butalelbun más habla es chedungun [...] si un no-mapuche está presente se habla castellano para que él entienda, pero si estamos reunidos todos nosotros hablamos chedungun no más".

"En el ngillatun generalmente se habla el chedungun, pero también se ha perdido un poco, porque yo me acuerdo como 20 años atrás, en Cauñicu el ngillatun la mayoría hablaba el chedungun, pero ahora ya no. No que no se hable, pero menos que antes [...]. No se puede hacer un ngillatun en castellano, no tiene ningún sentido".

6 El uso del mapudungun en reuniones comunitarias públicas, en festividades y celebraciones religiosas, si bien representa un empleo no regular de la lengua donde resulta poco probable que los menores aprendan el idioma (en el sentido de adquirir su competencia comunicativa) constituye un factor importante que suscita conciencia etnolingüística en el grupo. 


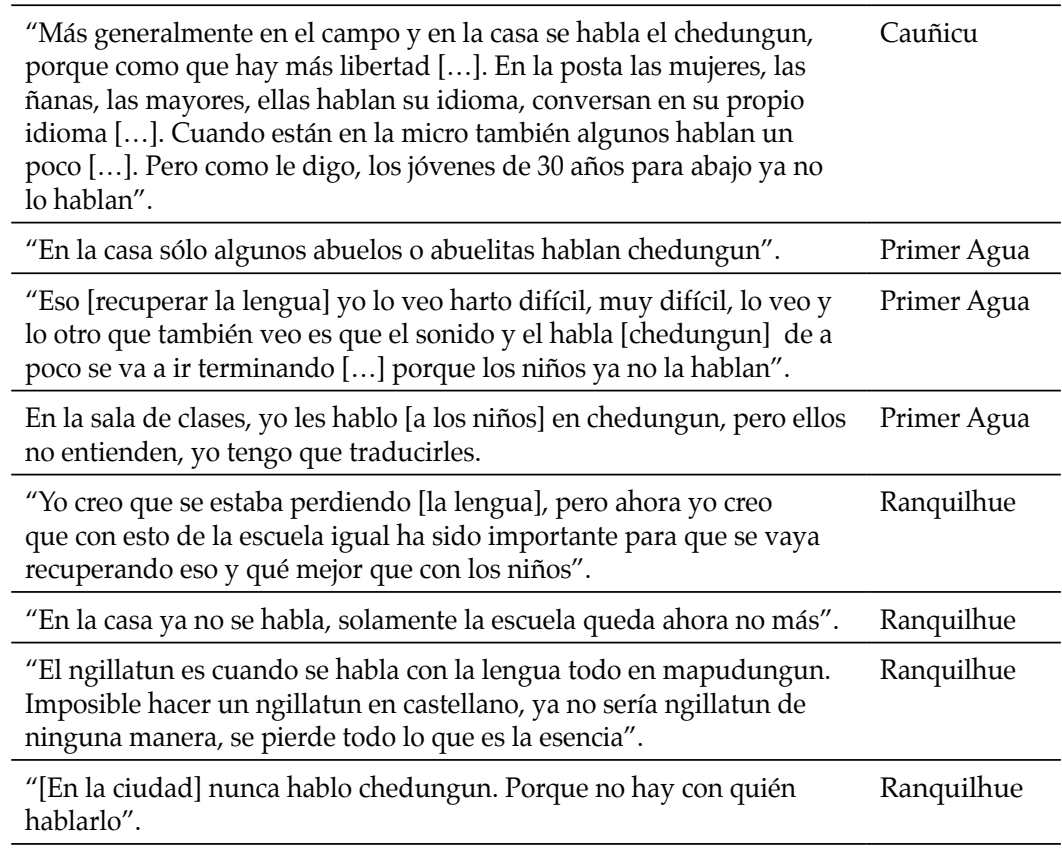

En líneas generales, y según los datos reportados por nuestros entrevistados, es posible sostener, como ya lo habían señalado los niños, que para los pewenches de Butalelbun y Cauñicu el empleo del chedungun es claramente mayoritario en la esfera familiar e íntima, aunque en Cauñicu la lengua mayoritaria ha comenzado a filtrase en ámbitos tradicionalmente reservados al vernáculo, provocando fenómenos de variación lingüística, especialmente entre miembros de las generaciones más jóvenes. Esto no sucede en Butalelbun, donde la preeminencia de la lengua trasciende al conjunto de la comunidad ${ }^{7}$, manteniendo de esta forma una posición sólida en los diferentes dominios de uso tradicionales. De este modo, se cumple en Butalelbun la premisa señalada por los especialistas: mientras más dominios de uso, mayor vitalidad lingüística (Blas Arroyo, 2008). En Cauñicu, los datos

7 Según pudimos constatar a través de conversaciones sostenidas con diferentes miembros de la comunidad, uno de los pocos lugares donde se usaba exclusivamente el castellano era en las celebraciones de la iglesia evangélica. El pastor que en ese entonces dirigía la ceremonia religiosa no pertenecía a la comunidad y no hablaba chedungun, por lo tanto toda la celebración se realizaba exclusivamente en castellano. 
muestran un proceso de desplazamiento ${ }^{8}$ en marcha del chedungun en beneficio del castellano.

En Tirúa, los asesores respaldan lo que informaron los escolares, en el sentido de que el empleo de la lengua originaria está asociada principalmente a dos ámbitos sociolingüísticos: las conversaciones familiares, cuando los interlocutores son ancianos o personas mayores, y las ceremonias religiosas tradicionales. Fuera de estos dominios, el uso del castellano es general, aunque en la escuela se ha comenzado a introducir el mapudungun de manera paulatina.

Con respecto a esto último, creemos que es importante tener en cuenta que el apoyo institucional a una lengua puede influir en su conservación o mantenimiento y, como se sabe, dos de las instituciones más importantes son la educación y la religión ${ }^{9}$ (Giles et al., 1977; Fasold, 1996; Serrano, 2011). Sin duda, la escuela puede actuar como impedimento o núcleo de desarrollo de una lengua, de modo que el hecho de que se emplee el mapudungun en el sistema educacional tiene una importancia capital. En estas localidades, según nos informan nuestros entrevistados, la escuela es hoy en día un escenario de uso y revitalización del idioma más relevante que el hogar. Esto tiene, en cierto modo, una interpretación positiva, ya que en la actualidad el mapudungun y lo mapuche se han incorporado en el currículum educativo, permitiendo el avance y la dignificación social de lo mapuche en la escuela (Olate y Henríquez, 2010).

8 Los datos fonético-fonológicos que hemos recogido en esta zona respaldan esta apreciación sociolingüística, ya que los escolares de Cauñicu muestran una significativa merma de la vitalidad fonológica del chedungun en comparación con los estudiantes de Butalelbun, presentando fenómenos de alternancia fonológica y procesos de transferencia del castellano más sistemáticos y abundantes que los presentes en Butalelbun (Henríquez, 20013; Henríquez y Salamanca, 2012).

9 Para los especialistas, la religión puede ser un factor unificador y a la vez separador en las comunidades minoritarias, y, en este caso, según las observaciones in situ, creemos que es unificador cuando, por ejemplo, en las comunidades mapuches se celebra el ngillatun y los miembros de la comunidad participan y en este escenario se habla sólo mapudungun. Es un factor de separación cuando los mapuches se niegan a "entrar" al ngillatun, porque participan de oficios religiosos occidentales. Esta situación es más usual en las familias que pertenecen a la iglesia evangélica, donde no sólo no se habla mapudungun, sino que muchas veces se prohíbe. Al respecto, la educadora tradicional de Ranquilhue nos comentó lo siguiente: "lo que pasa es que en las iglesias de repente se les prohibe participar en un ngillatun, se les prohibe andar con sus prendas, vestirse como tal [...] por eso yo creo que no se les permiten esas cosas. Esto ocurre más en la iglesia evangélica [...] yo por lo menos no he visto ningún evangélico que participe en el ngillatun". La situación que describe la educadora no es exclusiva de Ranquilhue, pues la hemos constatado, en mayor o menor grado, en todas las localidades. El problema sociolingüístico que trae consigo este hecho, al menos en Tirúa, es que el ngillatun es uno de los pocos espacios públicos tradicionales que van quedando, donde se practica mayoritariamente el mapudungun, y cuando las familias se restan de participar no les están dando la oportunidad a los niños de ser parte de este importante evento comunicativo donde el mapudungun es una lengua altamente funcional. Por lo demás, cuando se restringen las posibilidades de uso de la lengua minoritaria se contribuye a su desplazamiento (Serrano, 2011). 
La pérdida o reducción de ámbitos de uso de la lengua tiene consecuencias significativas no sólo para el mantenimiento de la lengua minoritaria (Fasold, 1996; Blas Arroyo, 2008; Serrano, 2011), sino para los procesos de pérdida lingüística en general (Crystal, 2001; Gimeno y Gimeno, 2003). Creemos que esto ha sucedido en Tirúa, ya que el desplazamiento de la lengua en el dominio íntimo y familiar ha provocado una fractura en la transmisión intergeneracional y, por ende, la disminución del número de hablantes jóvenes y la pérdida de competencia lingüístico-comunicativa en este grupo etario.

Por último, es relevante reiterar que la elección de una lengua en los contextos bilingües depende de la interacción de una serie compleja de factores, por lo que no puede explicarse completamente o adecuadamente atendiendo tan sólo a los rasgos situacionales (Dorian, 1981). En efecto, existen también factores psicológicos, culturales y lingüísticos implicados en este proceso, los cuales resultan determinantes en la decisión de los hablantes, por lo que su estudio en profundidad es de vital importancia.

\section{Conclusiones}

En Alto Bío-Bío se observa que el chedungun es altamente funcional en los diferentes espacios intracomunitarios. Esto ocurre principalmente en Butalelbun, donde la presencia de esta lengua en los dominios domésticos, íntimos e informales, constituye uno de los factores más explicativos de su alto grado de retención. En Cauñicu, en cambio, la lengua ancestral día tras día pierde terreno en diversos espacios comunicativos y, actualmente, el hogar y las celebraciones religiosas tradicionales constituyen los principales reductos para su empleo; pero, incluso en estos ámbitos, su uso disminuye considerablemente en las relaciones comunicativas entre miembros de generaciones más jóvenes. En esta comunidad existen familias que aún mantienen el mapudungun como la lengua del hogar; sin embargo, hay otras, en que el castellano es el medio principal de comunicación. Esta situación es relevante, pues, como se sabe, uno de los escenarios más habituales del desplazamiento lingüístico corresponde al abandono de la lengua minoritaria en el seno familiar.

En Tirúa, los datos muestran un cambio significativo en la distribución de usos y funciones de las lenguas en contacto. Así, las entrevistas $\mathrm{y}$ encuestas a nuestros colaboradores muestran un significativo 
aumento del castellano en diversos ámbitos intracomunitarios en desmedro del mapudungun, incluso en aquellos dominios domésticos e informales que tradicionalmente estaban reservados al vernáculo. En ambas comunidades hemos observado un significativo proceso de desplazamiento del mapudungun al interior de las familias, el que se hace evidente no sólo en la reducción de sus dominios, sino también en la escasez de hablantes entre las generaciones de adultos y jóvenes. Hoy en día, la lengua ancestral se encuentra fundamentalmente en manos de los ancianos y ha quedado relegada a los ámbitos de uso más formales y ceremoniales al interior de la comunidad. 


\section{Referencias}

Andersen, R. (1982). Determining the linguistic attributes of language attrition. En Lamber, R. and Freed, B. (Eds.), The loss of language skills. Rowley, Mass: Newsbury House Publishers.

Appel, René y Muysken, P. (1996). Bilingüismo y contacto de lenguas. Barcelona: Ariel.

Blas Arroyo, José Luis (2008). Sociolingüística del español. Desarrollos y perspectivas en el estudio de la lengua española en contexto social. Madrid: Ediciones Cátedra.

Crystal, D. (2001). La muerte de las lenguas. Cambridge: Cambridge University Press.

Dorian, N. (1977). The problem of the semi-speaker in language death. International Journal of the Sociology of Language, 12, 23-32.

- (1980). Language Shift in Community and Individual: The Phenomenon of the Laggard Semi-Speaker. International Journal of the Sociology of Language, 25, 85-94.

. (1981). Language Death. The Life Cycle of a Scottish Gaelic Dialect. Philadelphia: University of Pennsylvania Press.

Fasold, R. (1996). La Sociolingüística de la sociedad. Introducción a la sociolingüística. Madrid: Visor Libros.

Giles, H., Bourhis, R. y Taylor, D. (1977). Towards a theory of language in ethnic group relations. En H. Giles (Ed.), Language, Ethnicity and Intergroup Relations (pp. 307-348). London: Academic Press.

Fishman, J. (1971). Bilingualism in the Barrio. Bloomington: Indiana University Press. (1982). Sociología del lenguaje. Madrid: Ediciones Cátedra.

Gimeno, F. y Gimeno, M. (2003). El desplazamiento lingüístico del español por el inglés. Madrid: Ediciones Cátedra. 
Grinevald, C. (1997). Language contact and language degeneration. En F. Coulmas (Ed.), The Handbook of Sociolinguistics (pp. 257-270). Oxford: Basil Blackwell.

Henríquez, M. (2013). Vitalidad fonológica del mapudungun en escolares mapuches pewenches y lafkenches de la VIII región del Bío-Bío. (Tesis de Doctorado en Lingüística). Universidad de Concepción, Concepción, Chile.

Henríquez, M. (2014). Estado del mapudungun en comunidades pewenches y lafkenches de la región del Bío-Bío: el caso de los escolares. Revista RLA, 52 (2),13-40.

Henríquez, M. y Salamanca, G. (2012). Rasgos prominentes de la fonología segmental del chedungun hablado por escolares pewenches del Alto Bío-Bío. Revista Alpha, $34,152-172$.

Hernández, R., Fernández, C. y Baptista, P. (2003). Metodología de la investigación. México: McGraw-Hill.

Kirschner, C. (1996). Language attrition and the spanish-english bilingual: a case of syntactic reduction. Bilingual Review, 21 (2), 123-130.

Moreno Fernández, Francisco (2005). Principios de sociolingüística y sociología del lenguaje, $2^{\circ}$ edición actualizada. Barcelona: Ediciones Ariel.

Olate, Aldo y Henríquez, Marisol (2010). Actitudes lingüísticas de profesores mapuche de Educación Básica: vigencia y enseñanza del mapudungun en el contexto educativo. Literatura y Lingüística, 22, 103-116.

Olate, A., Alonqueo, P. y Caniguan, J. (2013). Interactividad lingüística castellano/mapudungun de una comunidad rural bilingüe. Alpha, 37, 265-284.

Saville Troike, M. (2005). Etnografía de la comunicación. Una introducción. Buenos Aires: Prometeo Libros.

Serrano, M. J. (2011). Sociolingüística. Barcelona: Ediciones del Serbal.

Siguan, M. (2001). Bilingüismo y lenguas en contacto. Madrid: Alianza Ensayo. 